\title{
A Review of Data-Driven Oil and Gas Pipeline Pitting Corrosion Growth Models Applicable for Prognostic and Health Management
}

\author{
Roohollah Heidary ${ }^{1}$, Steven A. Gabriel², Mohammad Modarres ${ }^{3}$, Katrina M. Groth ${ }^{4}$, and Nader Vahdati ${ }^{5}$ \\ 1,2,3,4 Center for Risk and Reliability, Department of Mechanical Engineering, University of Maryland, College Park, MD 20742, USA \\ heidary@umd.edu \\ sgabriel@umd.edu \\ modarres@umd.edu \\ kgroth@umd.edu \\ ${ }^{5}$ Department of Mechanical Engineering, Khalifa University of Science and Technology, Sas Al Nakhl Campus, Abu Dhabi, UAE 2533 \\ nvahdati@pi.ac.ae
}

\begin{abstract}
Pitting corrosion is a primary and most severe failure mechanism of oil and gas pipelines. To implement a prognostic and health management (PHM) for oil and gas pipelines corroded by internal pitting, an appropriate degradation model is required. An appropriate and highly reliable pitting corrosion degradation assessment model should consider, in addition to epistemic uncertainty, the temporal aspects, the spatial heterogeneity, and inspection errors. It should also take into account the two well-known characteristics of pitting corrosion growing behavior: depth and time dependency of pit growth rate. Analysis of these different levels of uncertainties in the amount of corrosion damage over time should be performed for continuous and failure-free operation of the pipelines. This paper reviews some of the leading probabilistic data-driven prediction models for PHM analysis for oil and gas pipelines corroded by internal pitting. These models categorized as random variable-based and stochastic process-based models are reviewed and the appropriateness of each category is discussed. Since stochastic process-based models are more versatile to predict the behavior of internal pitting corrosion in oil and gas pipelines, the capabilities of the two popular stochastic process-based models, Markov process-based and gamma process-based, are discussed in more detail.
\end{abstract}

\footnotetext{
Heidary et al. This is an open-access article distributed under the terms of the Creative Commons Attribution 3.0 United States License, which permits unrestricted use, distribution, and reproduction in any medium, provided the original author and source are credited.
}

\section{INTRODUCTION}

Corrosion is the main failure mechanism of oil and gas pipelines. Of all corrosion mechanisms, pitting corrosion is of most concern in pipelines because of the high rate at which pits can grow (Velázquez, Caleyo, Valor, \& Hallen, 2009). Failure data, provided in the literature, shows that $57.7 \%$ of oil and gas pipeline failures in Alberta, Canada between 1980 and 2005 (Papavinasam, 2013) and 15\% of all transmission pipeline incidents between 1994 and 2004 in the US were due to internal corrosion (Papavinasam et al., 2006). Moreover, $90 \%$ of corrosion failures of transmission pipeline sector in the US, between 1970 and 1984 were due to localized pitting corrosion (Papavinasam, 2013). Therefore, this review paper primarily discusses pitting corrosion growth prediction models applicable for PHM of oil and gas pipelines.

Despite significant research efforts in forecasting pitting corrosion, there are still many unanswered questions due to the highly stochastic nature of the pitting corrosion mechanism and a large number of dependent and independent influential parameters (Tarantseva, 2010) (Caleyo, Velázquez, Hallen, Valor, \& Esquivel-Amezcua, 2010). For example, parameters that may influence internal pitting corrosion are the $\mathrm{pH}$ value in the water phase, the water chemistry, the protective scale, the $\mathrm{CO}_{2}$ partial pressure, the amount of $\mathrm{H}_{2} \mathrm{~S}$, the effect of oil wetting, the metal alloy composition, the temperature, the multiphase flow, and the flow rate. Due to the large variations in these parameters, interdependencies between them, and also non-monotonic effects of some of them, there is a multitude of degradation 
paths for every single pit. In addition to this variation in degradation paths for each pit, there is usually more than one pit in a segment of a pipeline and each pit must be analyzed individually (by considering dependencies and correlations between pits) because the failure at each pit is equal to the failure of the whole pipeline. Figure 1 depicts an example of pitting corrosion on X70 carbon steel surface in a corrosive environment.

In comparison with conventional reliability analysis that mostly gives a population-based assessment, PHM approaches can handle corrosion complexity more efficiently, especially by utilizing fast developing information and inspection technologies that make it possible to have real-time data management and processing for each individual pipeline and also individual pit (Tsui, Chen, Zhou, Hai, \& Wang, 2015). Pipeline PHM approaches predict the remaining useful life (RUL) of a pipeline based on imprecise past and current degradation data gathered through some monitoring regime; this imprecision is due to uncertain inspection date. This estimation of RUL is vital in conditionbased maintenance by avoiding unnecessary maintenance and unpredicted failures (Imanian \& Modarres, 2017) (Rabiei, Droguett, Modarres, \& Amiri, 2015).

Most PHM methods rely on physics-of-failure (PoF)-based or data-driven based models (Imanian \& Modarres, 2017). PoF-based models have advantages in long-term damage behavior prediction, but since they are based on some approximations and simplifying assumptions when the degradation process is complex (e.g., pitting corrosion), it is difficult to estimate the model parameters and validate the results (An, Kim, \& Choi, 2015). However, studying PoFbased models is important to identify the root causes of pitting corrosion that can provide useful information for prognostic purposes. Because of the complexity and inherent randomness of pitting corrosion over time, probabilistic datadriven models are more suitable to describe pitting corrosion behavior especially when the results of modeling are used to perform reliability analysis (Shibata, 1996) (Valor, Caleyo, Alfonso, Rivas, \& Hallen, 2007) (Bazán \& Beck, 2013).

In probabilistic data-driven models, the knowledge about dependencies between pit depth and independent covariates, and also the uncertainties about the degrading process, are encapsulated in the inspection data. The extrapolated RUL prediction is valid and applicable as long as the resulting model from these inspection data is used for predicting RUL in pipelines with a similar operational condition. Among different probabilistic data-driven PHM approaches (Tsui et

\footnotetext{
${ }^{1}$ It worth noting that internal corrosion of oil and gas pipelines made from carbon steel is often referred to as "sweet $\left(\mathrm{CO}_{2}\right)$ corrosion". However other corrosive species such as hydrogen sulfide, $\mathrm{H}_{2} \mathrm{~S}$ (sour corrosion), organic acid, etc., might be involved in this corrosion process as well (Nešić, 2007). Among these corrosion species, presence of $\mathrm{H}_{2} \mathrm{~S}$, changes corrosion mechanism tremendously because of production of iron sulfide instead of iron
}

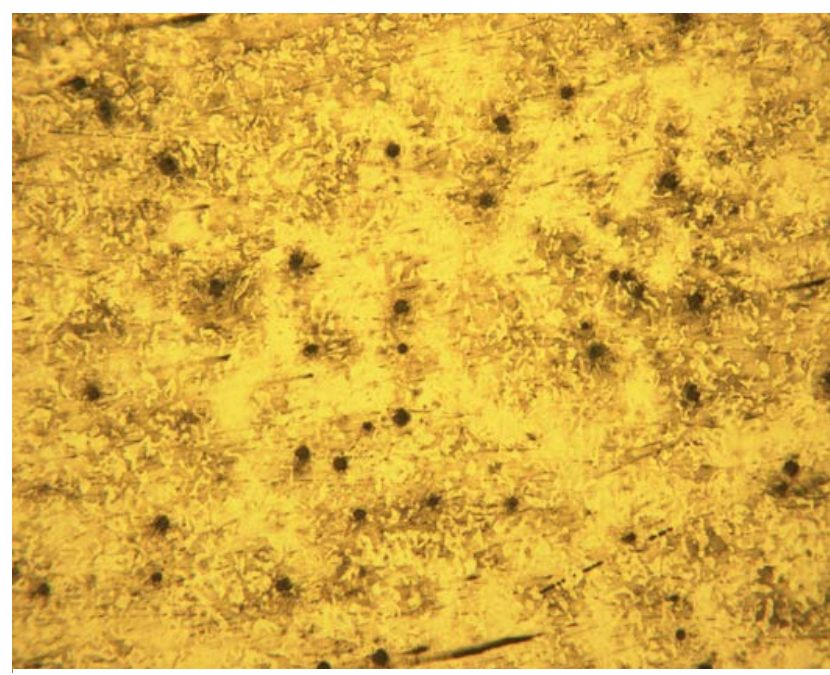

Figure 1. An example of pitting corrosion on X70 carbon steel surface in a corrosive environment (magnification scale: 200X) (Modarres \& Nuhi, 2010)

al., 2015) (An et al., 2015), this paper discusses regressionbased, gamma process-based, and Markov process-based models that are usually used to represent pitting corrosion process in oil and gas pipelines.

To emphasize the contribution of this review paper, the readers should make note of some other review papers (Papavinasam, 2013) (Papavinasam et al., 2006) (Tarantseva, 2010) (Nyborg, 2010) (Nešić, 2007) that categorize different corrosion rate models and modeling approaches. Key conclusions of these review papers will follow.

Nyborg (2010) compared the performance of fourteen uniform $\mathrm{CO}_{2}{ }^{1}$ corrosion rate models for oil and gas production systems by applying these models to some reliable data from some operating companies. Some of these models are empirical and were obtained by using empirical regression analysis. On the other hand, some of them are mechanistic that take the chemical, electrochemical or transport processes into account and some of them are a combination of these two approaches and are semi-empirical. Among all these models, just four of them have considered the localized corrosion (e.g. pitting corrosion, crevice corrosion). Nyborg (2010) concluded that none of these fourteen models significantly performed better than the

carbonate. Therefore, using sweet corrosion model, even by adding sulfide correction factor, will not give reliable results (Nyborg, 2010). Based on field corrosion data, $\mathrm{H}_{2} \mathrm{~S}$ is related to the occurrence of localized corrosion, however, the mechanism and location of happening are not well understood (Nešić, 2007). 
others for all cases and none of these models can claim better than $\pm 50 \%$ accuracy for a wide range of conditions. Two main factors that cause this variability are corrosion films and oil wetting effects modeling approaches. As it has been shown in (Nyborg, CO2 corrosion models for oil and gas production systems, 2010), those models that are mostly based on regression analysis and physics-of-failure analysis, cannot depict the inherent uncertainties in the corrosion process even for uniform corrosion. One reason for this inaccuracy is that these models mostly have considered level 1 uncertainty in Figure 2, which is related to lack of knowledge about the corrosion process (epistemic uncertainty), and they do not take into account the other three levels of uncertainty that are discussed later.

Nesic (2007) categorized available $\mathrm{CO}_{2}$ corrosion rate models for uniform internal corrosion of oil and gas pipelines into mechanistic models, empirical models and semiempirical models and discussed advantages and disadvantages of each category. Mechanistic models have a strong theoretical background and give accurate and physically realistic interpolation and extrapolation prediction when they are calibrated with a reliable experimental database. However, the main disadvantage of mechanistic models is that the prediction results might be unrealistic if many simplifying assumptions are used. Recalibrating mechanistic models by adding correction factors (to expand the range of application) leads to semi-empirical models. However, using semi-empirical models for extrapolation can result in having unreliable or unrealistic results. The third category is the empirical models (e.g., regression-based models, neural network-based models) that have very little or no theoretical background. These models perform very well within their calibration range but have to be used cautiously outside this range. Localized $\mathrm{CO}_{2}$ corrosion is considered briefly in (Nešić, 2007) as a process that is still not well understood and some recent works that have been done in this area are addressed.

Papavinasam et al. (2006), has reviewed different models that predict internal pitting corrosion of oil and gas pipelines. However, most of the models that are discussed in that review are addressing uniform $\mathrm{CO}_{2}$ corrosion rate.

In a nutshell, the above-mentioned models, including the probabilistic ones mostly correspond to level 1 in Figure 2 and they do not consider the other three levels of uncertainty.

Figure 2 shows the four hierarchical levels of uncertainty in degrading structures. Level 1, captures all model uncertainties (epistemic uncertainties) that are applicable to all points within a pipeline segment. The other three levels, apply to each local point in that segment; Level 2 indicates location-specific uncertainties that are related to uncertainties in known covariates (e.g., temperature, pressure, material properties) or aleatory effects due to unknown or omitted covariates (e.g., top of line corrosion that sometimes happens due to water condensation in natural gas pipelines); Level 3

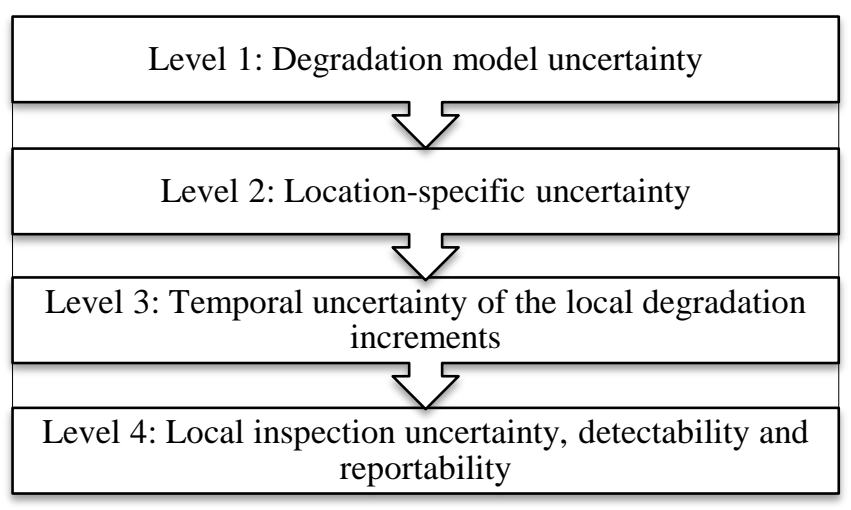

Figure 2. Hierarchical levels of uncertainty in degrading systems (modified) (Maes, Faber, \& Dann, 2009)

reflects the temporal uncertainty that models the difference between two defects, which even have the same load conditions and also in a similar location but can grow differently; and level 4 represents three kinds of inspection uncertainties (measurement error, probability of detection and reportability error) that need to be taken into account. Measurement error is a function of in-line inspection (ILI) device and measurement conditions, probability of detection (POD) is a function of the actual defect size, and reportability is a function of the lower detection threshold of the ILI device (Maes et al., 2009).

This paper defines an appropriate pitting corrosion degradation model for PHM analysis of oil and gas pipelines as a model that considers all of these four levels of uncertainty. In addition to the above-mentioned criteria, pitting corrosion rate has some characteristics all of which should be satisfied by an appropriate pitting corrosion degradation model. First, the pitting corrosion growth rate is depth-dependent (characteristic I; the corrosion rate of a deeper pit is greater than the corrosion rate of a shallower one) and second, the pitting corrosion rate is time-dependent (i.e., for a single pit the corrosion rate decreases over time (Nešić, 2007)(Valor, Caleyo, Alfonso, Velázquez, \& Hallen, 2013)) and this declining behavior follows a power law model with a less than one positive exponent (characteristic II) (Velázquez et al., 2009) (Ossai, Boswell, \& Davies, 2015) (Nuhi, Seer, Al Tamimi, Modarres, \& Seibi, 2011).

To the best of the authors' knowledge, there is no comprehensive review paper on pitting corrosion growth models applicable for prognostic and health management of oil and gas pipeline. This paper reviewed the commonly used pitting corrosion growth models; we focused on most highly cited and also more recently developed models. We then evaluated the published models by checking if they can model the above-mentioned characteristics and different uncertainty levels. 


\section{Probabilistic datA-DRIVEN MOdels}

Probabilistic data-driven based models can be classified into random-variable based and stochastic-process based models. The main difference between these two categories is that the latter one deals with the temporal variability of the degradation process, which leads to more realistic prediction (Zhang \& Zhou, 2013), while the former one does not consider the third level of uncertainty in Figure 2.

Figure 3 shows this classification and also the corresponding sub-classes for each class. These models are the most commonly used probabilistic data-driven ones that have been used in the literature to model pitting corrosion growth in the oil and gas pipelines. These models are explained in more detail in the following sections.

\subsection{Random Variable-Based Corrosion Growth Models}

The random variable-based corrosion growth models are the most common ones in the literature for reliability analysis of corroding pipelines (Zhang \& Zhou, 2013). These models consider corrosion uncertainty in terms of time-independent random variables. Linear and nonlinear random variablebased growth models are discussed below.

\subsubsection{Linear random variable corrosion growth model}

By having inspection data for at least two time instances, the growth rate for each pit depth can be estimated by the linear Eq.(1) and the probability distribution function of corrosion rate for a population of defects can be extracted accordingly.

$$
v_{d}=\frac{D\left(t_{2}\right)-D\left(t_{1}\right)}{t_{2}-t_{1}}
$$

where $v_{d}$ is the random variable that indicates the growth rate of a specific pit $(d), D\left(t_{2}\right)$ is the maximum depth of that pit $(d)$ at time $t_{2}$ and $D\left(t_{1}\right)$ is the maximum depth of that pit $(d)$ at time $t_{1}$. The randomness of the corrosion rate is due to the large variation in the depth of the pits caused by variations of the metal properties and the environmental conditions.

Linear random variable models are used commonly because they are simple and can be adjusted to limited corrosion data easily (i.e., only two sets of data, however, these models can be applied to one set of data as well, by assuming that stable pits start to grow from the beginning of corrosion process) (Bazán \& Beck, 2013). However, extrapolating this model over time overestimates the corrosion degradation and may give a conservative estimation of the reliability of the pipeline, because as mentioned before, the behavior of pitting corrosion growth follows a nonlinear power function with a positive exponent of less than one (Bazán \& Beck, 2013) (Ossai et al, 2015) (Nuhi et al., 2011). Another drawback of the linear models is that if they are projected backward, the pitting initiation time $t_{0}$ is often found to be negative which is physically meaningless (Bazán \& Beck, 2013). This model considers level 1 in Figure 2 nor the

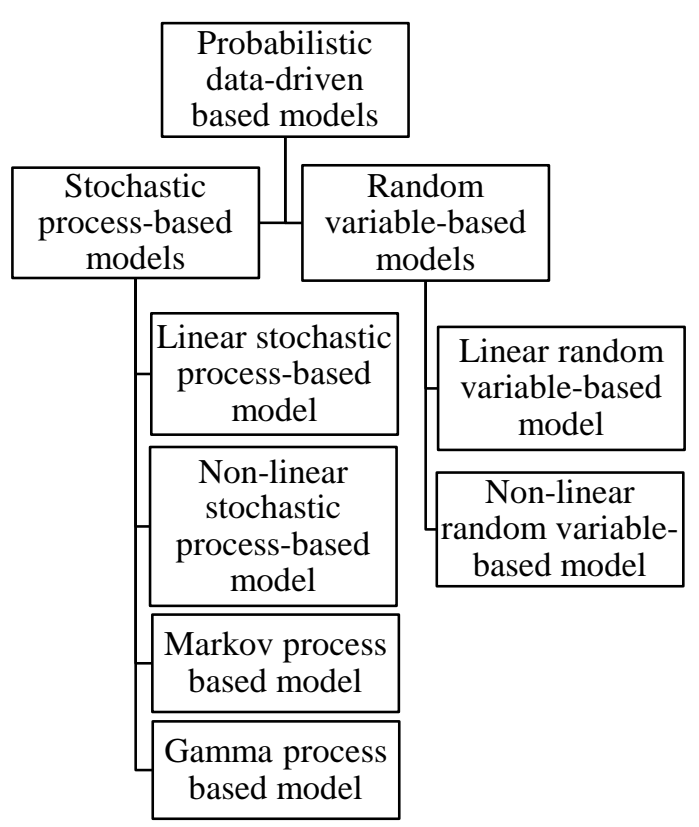

Figure 3. Breakdown of probabilistic data-driven based models for internal localized corrosion in pipelines

above-mentioned characteristics I and II. Temporal variability can be added to this model by using a Poisson square wave process that is explained in Section 2.2.1.

\subsubsection{Non-Linear random variable corrosion growth model}

As mentioned above, it is widely accepted that the pitting corrosion growth can be described by a power function with positive exponents of less than one (Bazán \& Beck, 2013) (Ossai et al., 2015) (Nuhi et al., 2011). Eq. (2) shows this model that is proposed in (Romanoff, 1957).

$$
D_{\max }(\mathrm{t})=k t^{\alpha}
$$

Considering the corrosion initiation time, a more accurate version of this model as shown in Eq. (3) is used by some other researchers (Velázquez et al., 2009) (Ossai et al., 2015).

$$
D_{\text {max }}(\mathrm{t})=k\left(t-t_{0}\right)^{\alpha}
$$

where $D_{\max }(\mathrm{t})$ is the maximum defect depth at time $t, t_{0}$ is the corrosion initiation time, $k$ is a proportionality factor and $\alpha$ is an exponent factor. Note that in external corrosion, $t_{0}$ represents the time that is required for coating damage plus the time period of effectiveness of cathodic protection, and in internal corrosion, $t_{0}$ represents the initiation time of stable pit growth (Valor et al., 2013). In an extension to the model in Eq. (3), Velazquez et al. (2009), performed a multivariate regression analysis to correlate the dependent variable $\left(D_{\max }\right)$ and independent variables (e.g. exposure time, soil and pipeline properties) for external pitting corrosion. They expressed $k$ and $\alpha$ as linear combinations of the soil and pipe variables as shown in Eq. (4). 


$$
\begin{gathered}
D_{\max }=k\left(t-t_{0}\right)^{\alpha} \\
=\left[k_{0}+\sum_{i=1}^{n} k_{i} x_{i}\right]\left(t-t_{0}\right)^{n_{0}+\sum_{j=1}^{m} n_{j} x_{j}}
\end{gathered}
$$

where $x_{i}$ is the $i_{t h}$ random predictor variable (e.g. $\mathrm{pH}$ ) and $k_{i}$ and $n_{j}$ are regression coefficients for this predictor.

Based on (Velázquez et al., 2009), the proportionality coefficient $k$ is mostly correlated to $\mathrm{pH}$, resistivity, dissolved ion concentrations, and redox potential. On the other hand, the exponent coefficient $\alpha$ is a function of water content, bulk density, coating type, and the pipe-to-soil potential.

In order to validate this model, Velázquez et al. (2009) plotted actual depth vs. predicted depth for 123 pits collected from another pipeline. Based on the visual examination of the plot, they concluded that the scatter of the predicted depth around the perfect correlation line was acceptable.

Ossai et al. (2015) used Eq. (5) to model internal pitting corrosion of sixty non-piggable oil and gas pipelines based on ten years of corrosion data. They assumed that the pitting initiation time is zero. The regression model that they used is shown in Eq.(5).

$$
D_{\text {max }}=k(t-0)^{\alpha}=\left(e^{\left(\gamma_{0}+\sum_{j=1}^{k} \gamma_{j} \gamma_{j}\right)}\right) t^{\alpha}
$$

Here $\gamma_{0}$ is the intercept, $\gamma_{j}$ is regression coefficient and $y_{j}$ is $j_{t h}$ predictor variable (i.e., operational parameters) that affects internal pitting corrosion. That study (Ossai et al., 2015) shows that $\mathrm{CO}_{2}$ partial pressure, flow rate, and chloride ion concentration are moderately correlated with maximum pit depth. In contrary, water cut, $\mathrm{pH}$ and sulfate ion concentration are weakly correlated with maximum pit depth.

Ossai et al. (2015) validated this model by calculating the root mean square percentage error (RMSPE) for the prediction data from three different pipelines with pits divided into four different pitting rate categories (low, moderate, high and severe). They calculated RMSPE for each combination with results ranging from 0.52 3.54, 0.59 7.26, 0.51 1.03 and 0.6 1.20 for low, moderate, high, and severe pitting corrosion rate category respectively. These ranges show the level of prediction accuracy of this model for each category.

This model considers level 1 in Figure 2 (epistemic uncertainty) and also characteristics II (having power law behavior) but it neither addresses the other levels in Figure 2 nor the characteristics I (dependency of the corrosion rate on the depth of the pit). Temporal variability can be added to this model by using a Poisson square wave process that is explained in Section 2.2.2.

\subsection{Stochastic-process based corrosion growth models}

The most commonly used stochastic processes that have been used to characterize the growth of corrosion defects are Markov process and gamma process (Zhang \& Zhou, 2013).
Two other stochastic processes, inverse Gaussian process (Zhang, Zhou, \& Qin, 2013) and Bayesian dynamic linear model (Zhang \& Zhou, 2014), also have been used for this modeling purpose and are discussed briefly at the end of the gamma process section. Before describing these processes, two other regression-based stochastic process-based models are presented.

\subsubsection{Linear stochastic process corrosion growth model}

As it was discussed in Section 2.1.1 and Section 2.1.2, random variable models do not consider the variability of corrosion growth over time (level 3 in Figure 2). To consider this temporal variability, Bazan and Beck (2013) modeled the defect growth rate as a Poisson square wave process (PSWP). Figure 4 shows a realization of a PSWP that represents the stochastic behavior of the defect growth rate (blue line). Moreover, this figure portrays a realization of the resulting stochastic defect size (red line), which is the accumulation of corrosion degradation at each random time interval. In this process, both pulse height $\left(Y_{i}\right)$ and pulse duration $\left(t_{i+1}-\right.$ $t_{i}$ ) are expressed as random variables. Pulse durations are characterized as independent and identically distributed (i.i.d) random variables that are exponentially distributed (Poisson process) and pulse heights (i.e., maximum pit depth growth rate) are characterized as i.i.d random variables that can be modeled by any strictly positive random variable distribution (e.g., the gamma distribution (Bazán \& Beck, 2013)). In this model, the maximum pit depth at each time instance can be estimated by using Eq. (6).

$$
\begin{gathered}
D_{\max }\left(t_{i+1}\right)=D_{\max }\left(t_{i}\right)+Y_{i}\left(t_{i+1}-t_{i}\right) \\
\text { for } i=0,1, \ldots, n
\end{gathered}
$$

where $n$ is the number of pulses, $D_{\max }(\mathrm{t})$ is maximum pit depth at time $t$, and $Y_{i}$ is the pulse height. Bazan and Beck (2013) used a data-fitting optimization algorithm to find out the parameters of this model (exponential distribution parameter for pulse durations, scale and shape parameter of gamma distribution for pulse heights) based on two sets of

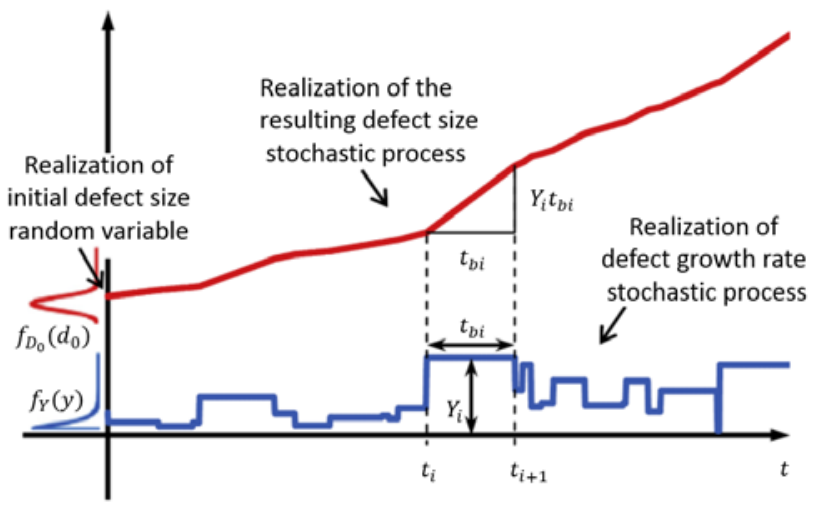

Figure 4. Linear stochastic process model (Bazán \& Beck, 2013) 
available inspection data. This model also (like the linear random variable model) has the limitation that backward extrapolation may lead to negative corrosion initiation time that is meaningless and violates the physics of the corrosion process.

\subsubsection{Non-Linear stochastic process corrosion growth model}

To add temporal variability to the model in Section 2.1.1, Bazan and Beck (2013) used PSWP with pulse heights $\left(Y_{i}\right)$ and durations $\left(t_{i+1}-t_{i}\right)$ according to Eq. (7) that shows the increment in defect size in each interval. Figure 5 shows a realization of this stochastic process.

$$
\begin{gathered}
D_{\text {max }}\left(t_{i+1}\right) \\
=D_{\text {max }}\left(t_{i}\right)+Y_{i}\left[\left(t_{i+1}-t_{0}\right)^{\alpha}-\left(t_{i}-t_{0}\right)^{\alpha}\right] \\
\text { for } i=0,1, \ldots, n
\end{gathered}
$$

In Eq. (7) $Y_{i}$ is the proportionality coefficient of maximum pit depth and operation parameters, $\alpha$ is the exponent coefficient, $n$ is the number of the pulses, and $t_{0}$ is the corrosion initiation time.

Having distributions of maximum pit depths at two time instances, parameters of this model were estimated by applying a data-fitting optimization algorithm (Bazán \& Beck, 2013). These parameters include the exponential distribution parameter (for pulse duration), scale and shape parameters of the gamma distribution (for pulse height), and scale and location parameters for lognormal distribution (for exponent factor). In contrast to linear models (Eq. (6)), the non-Linear stochastic process corrosion rate model (Eq. (7)) does not estimate the corrosion initiation time as a negative value.

Bazan and Beck (2013) calibrated these four models (linear random, non-linear random, linear stochastic and non-linear stochastic models) to the same set of corrosion data to explore the difference between them. They used actual corrosion data

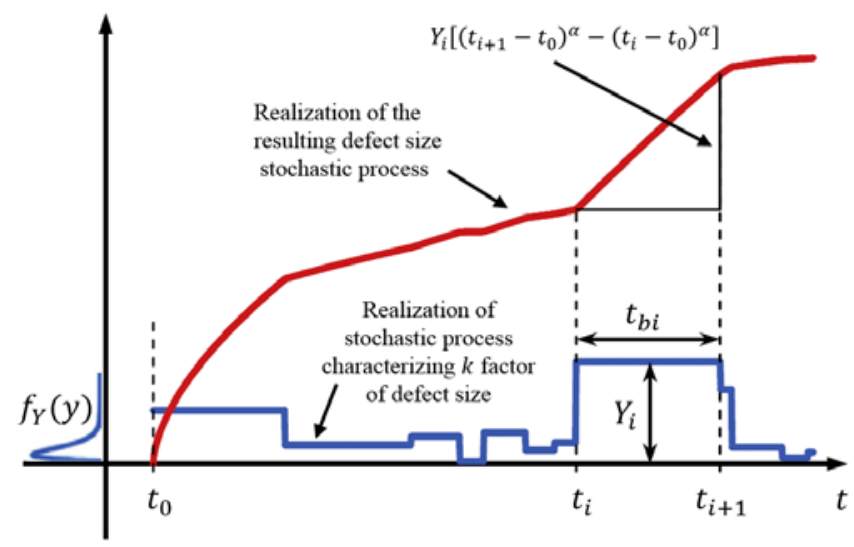

Figure 5. Non-Linear stochastic process model (Bazán \& Beck, 2013) in a pipeline collected for only two inspections. Therefore, without data on the third set of inspections, they could not evaluate the prediction capabilities of these four models. However, they showed that non-linear stochastic process model represents problem physics much better and it matches the available corrosion data reasonably well.

\subsubsection{Markov process-based corrosion growth models}

Markov processes have been used by many researchers to model corrosion process. The stochastic process $\{D(t), t \geq$ $0\}$ is a continuous-time Markov chain (Markov process), if for all $s, t \geq 0, d(u), 0 \leq u \leq s$, and nonnegative integers $i$ and $j$ :

$$
\begin{aligned}
P\{D(t+s)= & j \mid D(s)=i, D(u)=d(u), 0 \leq u \\
& <s\} \\
& =P\{D(t+s)=j \mid D(s)=i\}
\end{aligned}
$$

where $D(s)$, represents the condition (state) of the system at time $s$.

In other words, given that the system is in state $i$ at time $s$ $(D(s)=i)$, the future states $(D(t+s))$ do not depend on the previous states $(\mathrm{D}(u)=d(u), 0 \leq u<s)$. This is the socalled Markovian property and a continuous-time stochastic process is a Markov process if it satisfies the Markovian property. In addition, if $P\{D(t+s)=j \mid D(s)=i\}$ is independent of $s$, the Markov process is said to have homogeneous or stationary transition probability (Ross, 1996). In a Markov process, transition rate, $\lambda_{i}$, between states $i$ and $j$, are defined in such a way that the probability of transition between states $i$ and $j$ in the infinitesimal time interval $\delta t$, is $\lambda_{i} \delta t$ and the probability of more than one transition in this time interval is negligible. The Kolmogorov differential equation that represents this process is given in Eq. (9) (Ross, 1996) (Bhattacharya \& Waymire, 2009).

$$
\begin{gathered}
\frac{d P_{1}(t)}{d t}=-\lambda_{1} P_{1}(t) \\
\frac{d P_{i}(t)}{d t}=-\lambda_{i-1} P_{i-1}(t)-\lambda_{i} P_{i}(t), \\
i=2, \ldots, N \quad N=\text { Number of states }
\end{gathered}
$$

Here $P_{i}(t)$ represents probability of being in state $i$ at time $t$.

In Markov process-base corrosion rate models, the thickness of the pipeline is divided into $N$ finite states and presence of the maximum pit depth in each state at any point in time can be represented by a discrete random variable $D(t)$. The ultimate goal of the analysis is to predict probability of being in each state at each point in time $\left(P\{D(t)=i\}=P_{i}(t), i=\right.$ $1,2, \ldots, N)$. The important issue in these models is how to find the transition rate between states and how to correlate these transition rates to corrosion rates. In other words, these models try to find a valid set of transition rates between states by using available corrosion data for a specific pipeline to 
predict both the corrosion growth behavior of current pits and also new pits in the same or comparable pipelines. See (Ross, 1996) (Bhattacharya \& Waymire, 2009) for more details on Markov processes. In the following subsections, some main works that have been done in this area are presented.

Non-homogeneous Markov process corrosion growth model Provan and Rodriguez (1989) proposed a nonhomogeneous (transition rates are time-dependent) Markov process model to describe the growth of the maximum pit depth over time for a specific system for the first time. The gist of this model is the proposed non-homogeneous transition rate relationship shown in Eq. (10).

$$
\lambda_{j}(t)=\frac{\lambda j(1+\lambda t)}{1+\lambda t^{k}} \quad j=1,2, \ldots, n
$$

where $\lambda_{j}$ is the transition rate from state $j$ to state $j+1, t$ is exposure time, and positive values $\lambda$ and $k$ are the parameters of the pitting corrosion system. As mentioned before, the thickness of the pipeline is divided into a discretized space of states and being in each state indicates that the maximum depth of the pit is in that state. This model satisfies characteristic I and II that were mentioned previously. In other words, for greater $j$ (i.e., pit depth is in the deeper state), $\lambda_{j}$ is greater. Also for a constant $j$, transition rate (which is proportional to corrosion rate (Valor et al., 2007)) decreases over time (as long as $k$ is greater than 1 , which is the case of two examples that have been used in (Provan \& Rodriguez III, 1989)). To determine the parameters of the pitting corrosion system ( $\lambda$ and $k$ ), Provan et al. (1989) used an iteration procedure to find the proper values of $\lambda$ and $\mathrm{k}$ (which depend on the selected number of the states) that give the closest fit to their own experimental corrosion data for stainless steel (Provan \& Rodriguez III, 1989) and also corrosion data for aluminum given in (Aziz, 1956). To validate their model, Provan and Rodriguez (1989) visually compared the actual and predicted probability histograms of maximum pit depth at different points in time and concluded that their results give ample confidence in their proposed approach. The main drawback of this model (Eq. (10)) is that there is no physical meaning behind it and also there is no explanation about how to use this model for more than one pit (Valor et al., 2007). Also, this model does not address level 2, 3 and 4 uncertainties in Figure 2.

Non-homogeneous Poisson process for pit initiation and non-homogeneous Markov processes for pit growth Hong (1999) proposed a model in which pit initiation was modeled by a non-homogeneous Poisson process (which is a valid assumption because most of the pits are generating at the beginning of the corrosion process (Aziz, 1956), therefore pit initiation times are not homogeneously distributed). Also, pit growth process was modeled by a non-homogeneous Markov process. To find a closed-form solution for the Kolmogorov differential equation (Eq. (9)), first, the author has assumed homogeneity for both pits initiation times (homogeneous
Poisson process) and pits growth process (homogeneous Markov process) and then the time dependency that causes non-homogeneity was modeled by using the so-called timecondensation method (Bogdanoff, Kozin, \& Saunders, 1985). To do this transformation, the variable $t$ (that represents time) in the homogeneous equations is replaced by $t^{\beta}$, in which $\beta$ is a constant of the pitting corrosion that can be obtained by minimizing the errors of observed and predicted mean values of the maximum pit depths.

This model (Eq. (11)) gives the probability $\left(\theta_{i}(t)\right)$ that maximum pit depth be in a state less than or equal to state $i$ at time $t$ by considering all pits that have been generated in time interval $[0, t]$.

$$
\begin{gathered}
\theta_{i}(t)= \\
\exp \left(-v t^{\beta}(1-\right. \\
\left.\left.\frac{1}{\lambda t^{\beta}} \sum_{j=1}^{i} \frac{\gamma\left(j, \lambda t^{\beta}\right)}{(j-1) !}\right)\right), \quad i=1, \ldots, n-1
\end{gathered}
$$

where $v$ is pit generation rate, $\beta$ is the model parameter, $\lambda$ is the growth rate, $n$ is the number of discretized states, and $\gamma\left(j, \lambda t^{\beta}\right)$ is an incomplete gamma function.

To validate this model, Hong (1999) compared the mean of actual and predicted values of maximum pit depth visually. But Hong did not discuss any validation against additional data.

One drawback of Hong's model is that the proposed probability distribution of maximum pit depth is not a Gumbel distribution as it would be expected according to the published results in the literature (Valor et al., 2007); because it is well-known that extreme-value analysis using the Gumbel distribution is the most successful application in statistical analysis to predict the maximum pit depth in a large area by using a small number of samples within a small area (Shibata, 1996). Another drawback is that the results of the model depend on the number of states (Valor et al., 2007). In addition, it does not consider level 2, 3 and 4 uncertainties in Figure 2.

Non-homogeneous Linear Pure Birth Markov Process This model (Valor et al., 2007) (Caleyo, Velázquez, Valor, \& Hallen, 2009) proposes, for the first time, a methodology to link pit initiation and pit growth stages for multiple pits. For pit initiation, the Weibull distribution is used by interpreting the initiation time of each pit as the time to the first failure of a part of a system (Ascher \& Feingold, 1984). A continuoustime, non-homogeneous linear pure birth Markov process was used to model temporal non-homogeneity of pit evolution. In this process, transition rates from one state to another satisfy the forward Kolmogorov Equation (Ross, 1996) (Bhattacharya \& Waymire, 2009) with the following equation. 


$$
\lambda_{j}(t)=j \lambda(t)
$$

where $\lambda_{j}(t)$ represents the transition rate between the $j_{t h}$ to the $(j+1)_{t h}$ state during the time interval $[t+\delta t]$. $\delta t$ is an arbitrarily small unit of time that the probability of more than one transition is negligible. Since $\lambda_{i}(t)>\lambda_{j}(t)$ for $i>j$, characteristic I is already satisfied.

An advantage of using linear pure birth Markov process is that it has a closed-form solution for the transition probability from the $m_{t h}$ state to the $n_{t h}$ state in the interval $\left(t_{0}, t\right)$. This closed-form solution for this process that represents the negative binomial distribution is shown in Eq. (13) (Caleyo et al., 2009).

$$
\begin{gathered}
P_{m, n}\left(t_{0}, t\right) \\
=\left(\begin{array}{c}
n-1 \\
n-m
\end{array}\right) e^{-\left\{\rho(t)-\rho\left(t_{0}\right)\right\} m}\left(1-e^{-\left\{\rho(t)-\rho\left(t_{0}\right)\right\}}\right)^{n-m}
\end{gathered}
$$

where

$$
\rho(t)=\int_{0}^{t} \lambda(\tau) d \tau
$$

According to this equation by having $\lambda(t), \rho(t)$ can be estimated and subsequently $P_{m, n}\left(t_{0}, t\right)$ can be calculated by Eq. (13) and by having the initial pit depth distribution $\left(p_{m}\left(t_{0}\right)\right)$, the probability of being in each state at each point in time $\left(p_{n}(t)\right)$ can be estimated according to Eq. (14).

$$
p_{n}(t)=\sum_{m=1}^{n} p_{m}\left(t_{0}\right) p_{m, n}\left(t_{0}, t\right)
$$

The gist of this model is the proposed approach to find the transition rate $\lambda(t)$ based on the estimated corrosion growth model. This model is based on this assumption that the mean of the stochastic process (linear pure birth Markov process (Eq. (16)) can be assumed to be equal to the mean of the deterministic damage process (Eq. (3)). This assumption is valid for some processes under specific assumptions that are given in (Cox \& Miller, 1965). By equating these two means, $\lambda(t)$ can be obtained from Eq. (15).

$$
\lambda(t)=\frac{\alpha}{t-t_{0}}
$$

where $\alpha$ and $t_{0}$ are the exponent coefficient and corrosion initiation time in the power law model (Eq. (3)).

The mean of the linear growth Markov process can be estimated by Eq. (16).

$$
M(t)=\sum_{m=1}^{n} m \cdot p_{m}(t)
$$

Where $n$ is the number of the states and $p_{m}(t)$ is the probability of being in state $m$ at time $t$.

In this approach, there is no limitation on the number of discretized states because there is a closed-form solution for this model. However, there are two important unanswered questions about this model that have been mentioned by the authors of this paper themselves. First, the validity of the assumption of equating stochastic and deterministic means for the case of pitting corrosion and second, the applicability of this model for different kind of pit populations.

An important question that has been answered by this model in (Valor, Caleyo, Alfonso, Rivas, \& Hallen, 2007) is that how to use Eq. (13) for multiple pits with different pit initiation times. By assuming that $m$ pits initiate and grow independently and also assuming that all of them are in state 1 at initiation time, the probability that the deepest pit is in a state less than or equal to state $i$ at time $t$ can be estimated by Eq. (17).

$$
\theta(i, t)=\prod_{k=1}^{m}\left\{1-\left(1-e^{-\left\{\rho\left(t-t_{k}\right)\right\}}\right)^{i}\right\}
$$

Where pit initiation process is considered by parameters $t_{k}$ and pit growth process is considered by $\rho(t)$.

Valor et al. (2007) showed that for large $m$, this cumulative distribution function (Eq. (17)) follows a Gumbel distribution and for a special case (assuming pit initiation times are equal for all pits) they found a lower bound for $m$ as an important parameter when pit initiation and growth are combined in their proposed model.

The parameters of the proposed model $\left(t_{k}, \rho(t)\right.$ and $\left.m\right)$ can be obtained by minimizing a total error function $E_{T}$ given in Eq. (18).

$$
E_{T}=\sum_{i=1}^{N}\left(\sqrt{\left(\mu_{e}^{i}-\mu_{p}^{i}\right)^{2}}+\sqrt{\left(\sigma_{e}^{i}-\sigma_{p}^{i}\right)^{2}}\right)
$$

Where $\left(\mu_{p}^{i}, \sigma_{p}^{i}\right)$ and $\left(\mu_{e}^{i}, \sigma_{e}^{i}\right)$ are the mean value and variance of the $i_{t h}$ predicted and experimental extreme value distribution; respectively.

Valor et al. (2007) validated this model by calculating the mean root-square error (MRSE). They used experimental data published by Aziz (1956), Provan and Rodriguez (1989), Melchers (2004), Strutt, Nicholls, \& Barbier (1985), and showed that the results of their proposed model using those experimental data are better (lower MRSE) than reported results in the those works. This model had the most extensive validation in comparison with the other workes that are reviewed in the current paper.

This model considered characteristic I and II. Also, this model considers pit initiation process, pit growth process, and multiple independent pits growth. However, it does not consider level 2, 3 and 4 uncertainties in Figure 2.

Another drawback of Markov process-based models is that it is not straightforward to update these models by Bayesian inference in case of new imperfect ILI data (Zhang \& Zhou, 2014). 


\subsubsection{Gamma process-based corrosion growth models}

The gamma process is a stochastic process with independent, non-negative, gamma distributed increments. Mathematically speaking, a gamma process with shape function $\alpha(t)$ and scale parameter $\beta$ is a stochastic process $\{D(t), t \geq 0\}$ with the following properties:

$\alpha(t)>0$ is a non-decreasing, right-continuous, real-valued function for $t \geq 0$ and $\alpha(0)=0$

- $\beta>0$

- $D(0)=0$ with probability 1

- $D(\tau)-D(t) \sim G a(\alpha(\tau)-\alpha(t), \beta)$ for all $\tau>t \geq 0$;

- $D(t)$ has independent increment

Where $\mathrm{Ga}$ indicates gamma distribution with following probability density function.

$$
G a(d \mid \alpha, \beta)=\frac{\beta^{\alpha}}{\Gamma(\alpha)} d^{\alpha-1} \exp (-\beta d) \cdot I_{(0, \infty)}(d)
$$

where $I_{(0, \infty)}(d)=1$ for $d>0$ and zero otherwise and $\Gamma(\alpha)=\int_{t=0}^{\infty} t^{\alpha-1} e^{-t} d t$ is the gamma function (Van Noortwijk, 2009).

Due to the monotonic increasing nature of the gamma process, it is an appropriate process for degradation mechanisms such as wear, fatigue, corrosion, creep, etc. Also, mathematical tractability is another advantage of this process. Using this process implies that the defect size is always increasing when there is no maintenance (Castro, Caballé, \& Pérez, 2015) (Frangopol, Kallen, \& Van Noortwijk, 2004).

Van Noortwijk (2009) published a comprehensive survey of the application of the gamma process in maintenance. The following briefly explains two examples of those works that have used the gamma process to model the pipeline corrosion defect growth.

Maes et al. (2009) proposed a hierarchical Bayes framework (Figure 6) to model pipeline defect growth subject to ILI uncertainty. This framework can incorporate new inspection data and update the corrosion growth model accordingly.

Different levels of uncertainty are considered in this work as follows. Level 4 in Figure 2 is related to inspection uncertainties. These uncertainties can be categorized to measurement error, detectability, and reportability error. In (Maes et al., 2009) measurement errors are assumed to be normally distributed with means equal to zero and known location dependent variances (that might be correlated to the other locations (level 2 and 4 in Figure 2)). Eq. (20) shows the relationship between measured size, $D_{M, j, i}$, and actual true degradation, $D_{j, i}$, for the $j_{t h}$ defect at $i_{t h}$ inspection time.

$$
D_{M, j, i}=D_{j, i}+\varepsilon_{j, i}
$$

Where $\varepsilon_{j, i}$ is the measurement error for a specific inspection device and measurement conditions at a given location that is usually correlated to inspection device bias and interpretation algorithm.

To model probability of detection (POD) that depends on the size of the defect, a detection indicator variable, $\left(D I_{j, i}\right)$, that follows Bernoulli distribution is defined according to Eq. (21). Then the observable degradation, $\left(D_{O, j, i}\right)$, would be the product of this detection indicator $\left(D I_{j, i} ;\right.$ with $D I_{j, i}=$ 1 corresponding to successful detection) and the uncertain measurement due to sizing error, $\left(D X_{M, j, i}\right)$ (Eq. (22)).

$$
D I_{j, i} \mid P D\left(D_{j, i}\right) \sim \text { bernoulli }\left(P D\left(D_{j, i}\right)\right)
$$

Where PD indicates probability of detection.

$$
D_{O, j, i}=D I_{j, i} \cdot D_{M, j, i}
$$

The reportability factor (that represents lower detection threshold of the ILI device) is defined as a binary indicator random variable, $\left(R_{j, i}\right)$ (Eq. (23)). Production of this factor to the observed degradation measurement, $\left(D_{O, j, i}\right)$, gives the degradation value reported by the inspection device (Eq. (24)).

$$
\begin{gathered}
R_{j, i}\left(D_{O, j, i}\right) \\
=\left\{\begin{array}{lll}
0 & \text { if } \quad D_{O, j, i}<I L I \text { device threshold } \\
1 & \text { if } \quad D_{O, j, i} \geq I L I \text { device threshold }
\end{array}\right. \\
D_{R, j, i}=R_{j, i} \cdot D_{O, j, i}
\end{gathered}
$$

Temporal uncertainties (level 3 in Figure 2) are modeled by considering the actual degradation at each inspection time as the summation of actual degradation at the previous inspection time and degradation increment between these two inspections. Because of two reasons, gamma process is an appropriate process to model the degradation increment behavior (Eq. (25)); independent degradation increments assumption (Van Noortwijk, 2009) and restriction that such increments must be positive.

$$
\Delta D_{j, i} \mid \Delta \alpha_{j, i}, \beta_{j} \sim \operatorname{gamma}\left(\Delta \alpha_{j, i}, \beta_{j}\right)
$$

Where $\Delta \alpha_{j, i}$ is the shape parameter and $\beta_{j}$ is the scale parameter of the gamma distribution given in Eq. (19).

The shape parameter reflects the time dependency of the physics of the degradation process (level three of the uncertainty in Figure 2). By selecting a proper functional form for the shape parameter, different degradation processes can be modeled. Power law function (Eq. (26)) is a versatile function that can represent constant, increasing or decreasing degradation rate based on the exponent of the model.

$$
\Delta \alpha_{j, i}=\theta_{1}\left\{\left(t_{j, i-1}+\Delta t_{j, i-1}\right)^{\theta_{2}}-\left(t_{j, i-1}\right)^{\theta_{2}}\right\}
$$

Where $\theta_{1}$ and $\theta_{2}$ are degradation model's parameters that are related to epistemic uncertainty. $\theta_{2}>1$ stands for an 
increasing degradation process, $\theta_{2}<1$ expresses a decreasing degradation process, and $\theta_{2}=1$ represents a constant degradation process.

The scale parameter of the gamma process is a positive location specific parameter that reflects the heterogeneity of the defects between the locations (level 2 of uncertainty in Figure 2). The location heterogeneity is represented by $z_{j}, k$ and $\xi_{j} . z_{j}$ are local covariates (e.g., pressure, temperature, $\mathrm{pH}), k$ is a vector of cause and effect regression coefficients associated with $z_{j}$ and $\xi_{j}$ are local aleatory effects that cannot be explained by defined covariates (e.g. top of line corrosion).

$$
\beta_{j}=\exp \left\{\left(z_{j}\right)^{T} k+\xi_{j}\right\}
$$

Figure 6 summarizes this hierarchical Bayesian framework. In this figure, $\delta_{1}$ and $\delta_{2}$ are prior distribution Parameters for local aleatory effect model. According to this framework, by assuming prior probability density functions for system-wide parameters $\left(\theta_{1}, \theta_{2}, \delta_{1}, \delta_{2}, k\right)$, the actual true degradation, $D_{j, i}$, for $j_{t h}$ defect at $i_{t h}$ inspection time can be predicted. Then, as soon as inspection data $\left(D_{R, j, i}\right)$ become available (by knowing
As it was explained briefly, this hierarchical framework can model different level of uncertainties in the degradation systems and also characteristic II of pitting corrosion can be modeled by selecting proper values for $\theta_{1}$ and $\theta_{2}$. However, characteristic I is not addressed in this model directly.

Zhang and Zhou (2013) modified the above framework by considering corrosion initiation time in their model and used homogeneous gamma process $\left(\theta_{2}=1\right)$ to characterize the growth of the depth of corrosion defects. Zhang and Zhou (2013) used ILI data obtained in 2000, 2004 and 2007 for 62 defects to estimate their model parameters and they validated their model by comparing the defects' actual depths (obtained after excavation and field measurement) in 2010 with the corresponding depth predicted by their proposed growth model. This validation shows that $90 \%$ of the predicted depths fall within the region bounded by the two lines representing actual depth $\pm 10 \%$ pipe wall thickness.

Finally, two other works that used the similar Bayesian framework are discussed briefly. Zhang et al. (2013) used this framework by considering inverse Gaussian process (IGP) instead of homogeneous gamma process (HGP) to characterize the growth of the depth of corrosion defects. By

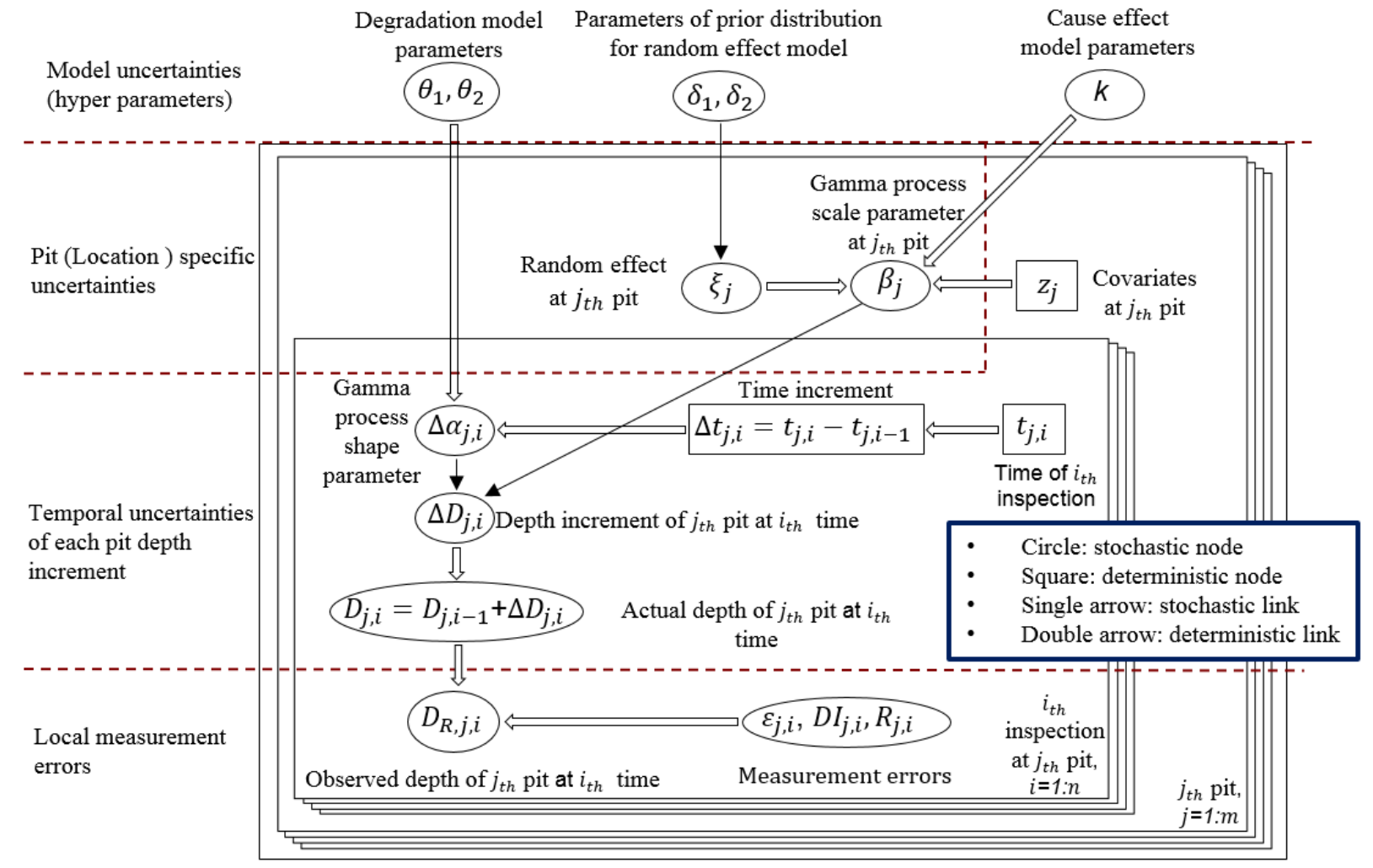

Figure 6. Hierarchical Bayes framework for heterogeneous degradation, modified from (Maes, Dann, Breitung, \& Brehm, 2008)

measurement error for each location and inspection time), the model's parameters can be updated by using a Bayesian updating simulation techniques such as Markov chain Monte Carlo (MCMC). applying this model to the same set of ILI corrosion data, they concluded that the predictions of the IGP-based model are negligibly different from those of the HGP-based model, but significantly better than random variable-based models. In 
another work, Zhang and Zhou (2014) applied a similar Bayesian framework by using a Bayesian dynamic linear model (BDLM) and compared the results with their previous results based on HGP and IGP. They showed that the absolute difference between predicted depths and corresponding field-measured depth is less than or equal to $10 \%$ pipe wall thickness for about $92 \%$ of the defects for BDLM, while this value for IGP and HGP is about $90 \%$ of the defects. This model is validated against a small number of corrosion data and it needs to be validated with larger data set to be able to be used it in practical application (Zhang \& Zhou, 2014).

\section{DisCUSSIONS}

Now we address the question of "when should each model be used?” To answer this question, two criteria are defined: appropriateness and practicality. As it is explained in Section 1 , an appropriate pitting corrosion growth model should consider four levels of uncertainty and also two well-known characteristics of pitting corrosion growing behavior: depth and time dependency of pit growth rate. The practicality criterion, indicates the level of knowledge that is required to perform each model. Table 1 summarizes this evaluation for those models that are discussed in this paper.

The first category in this table corresponds to linear random variable-based model which is the simplest probabilistic approach and also is the most commonly used approach in the industry that usually overestimates the pit growth rate. The second category corresponds to non-linear random variable-based model which is the one that considers the well-known non-linear behavior (power law function with a less than one positive exponent) of pitting corrosion process. None of these random variable-based models consider spatial heterogeneity, temporal variation, and measurement errors. They also do not take into account the depth dependency of the pitting corrosion rate.

The third and fourth categories correspond to linear and nonlinear stochastic process-based models that consider the temporal variability of pitting corrosion process. As it was discussed in Sections 2.2.1 and 2.2.2, these models are combinations of PSWP and linear and non-linear random variable-based models. The two stochastic process-based models also do not consider spatial heterogeneity and measurement errors. They also do not take into account the depth dependency of the pitting corrosion rate.

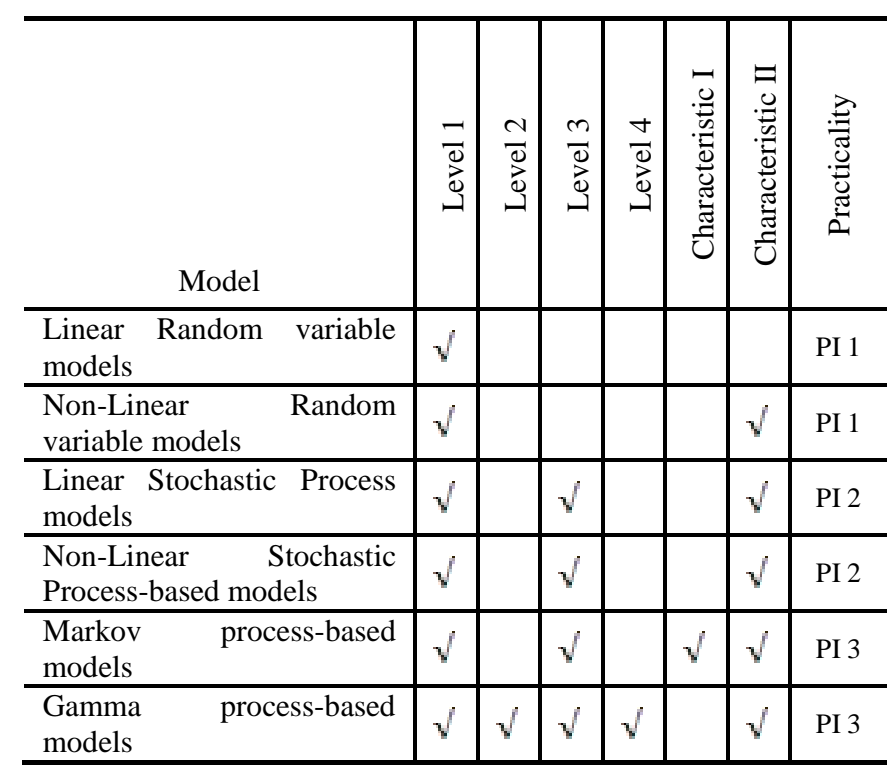

Table 1: Evaluation of different commonly used probabilistic data-driven pitting corrosion growth models in oil and gas pipelines

The fifth category corresponds to Markov process-based models. In these models, the main issue involves extracting proper transition rates between states. Markov process basedmodels consider epistemic uncertainty, temporal variability, non-linearity and also depth dependency of the pitting corrosion rates. However, the spatial heterogeneity and measurement uncertainties are not addressed properly in Markov process-based models and it is not straightforward to update these models by Bayesian inference in case of new imperfect ILI data.

The last category of this table corresponds to the gamma process-based models which are the most versatile models that can address different levels of uncertainties. Besides, they can model the non-linearity in the pitting corrosion growth process. These models can also be updated properly by Bayesian inference in case of new imperfect ILI data. However, depth-dependency has not been considered directly in these models. In the first six columns of Table 1 appropriateness of the abovementioned categories are evaluated.

The last column of Table 1 is allocated to practicality criterion. In this column, PI stands for Practicality Index. PI 1 is assigned to the first two categories and it means that these models can be developed by the common field engineers that are familiar with regression analysis using common application tools like Excel. PI 2 is assigned to the next two categories and it means that these models need more advanced knowledge of statistics such as PSWP. PI 3 is assigned to the last two categories and it needs a deep understanding of Markov process and gamma process. 


\section{CONCLUSION}

This paper reviews various pitting corrosion degradation models for PHM analysis. Degradation model is a key element of the PHM approach to predict the remaining useful life of a degrading system. Despite a large number of studies that have tried to find a comprehensive pitting corrosion growth model (degradation model), there is no universally accepted model that is able to predict the pitting corrosion growth properly for all occasions. Among available physicsof-failure and data-driven based pitting corrosion growth models, this review paper focused on the latter as they are more suitable to describe pitting corrosion behavior because of the complexity and inherent randomness of pitting corrosion over time. The reason is that pitting corrosion process is a stochastic process that depends on a large number of dependent and independent factors (epistemic uncertainties); moreover, this process has temporal and spatial heterogeneity; also, inspection uncertainties (measurement errors, probability of detection and reportability errors) add another level of uncertainty to the pitting corrosion growth estimation.

In addition to these different levels of uncertainties, an appropriate pitting corrosion growth model must be able to take into account the two other well-known characteristics of pitting corrosion; the corrosion rate of a deeper pit is greater than the corrosion rate of a shallower one, and for a single pit, the corrosion rate declines over time following a power-law function with a less than one positive exponent. This paper discussed the appropriateness of some probabilistic datadriven based models that are commonly used to predict pitting corrosion growth. In addition to the appropriateness, the practicality of these models is also discussed in this paper.

\section{ACKNOWLEDGEMENTS}

This work is being carried out as a part of the Pipeline System Integrity Management Project, which is supported by the Petroleum Institute, Khalifa University of Science and Technology, Abu Dhabi, UAE

\section{REFERENCES}

An, D., Kim, N. H., \& Choi, J. H. (2015). Practical options for selecting data-driven or physics-based prognostics algorithms with reviews. Reliability Engineering \& System Safety, 133, 223-236. doi:10.1016/j.ress.2014.09.014

Ascher, H., \& Feingold, H. (1984). Repairable systems reliability: Modeling, inference, misconceptions and their causes. New York: CRC Press/Marcel Dekker, Inc.

ASTM G46-94. (2005). Standard guide for examination and evaluation of pitting corrosion.

Aziz, P. (1956). Application of the statistical theory of extreme values to the analysis of maximum pit depth data for aluminum. Corrosion, 12(10), 35-46. doi:10.5006/0010-9312-12.10.35
Bazán, F. A., \& Beck, A. T. (2013). Stochastic process corrosion growth models for pipeline reliability. Corrosion Science, $\quad 74, \quad 50-58$. doi:10.1016/j.corsci.2013.04.011

Bhattacharya, R. N., \& Waymire, E. C. (2009). Stochastic processes with application. New York: Wiley.

Bogdanoff, J. L., Kozin, F., \& Saunders, H. (1985). Probabilistic models of cumulative damage. New York: John Wiley \& Sons.

Caleyo, F., Velázquez, J. C., Hallen, J. M., Valor, A., \& Esquivel-Amezcua. (2010). Markov chain model helps predict pitting corrosion depth and rate in underground pipelines. International Pipeline Conference (pp. 573581). Alberta,Canada: American Society of Mechanical Engineers.

Caleyo, F., Velázquez, J. C., Valor, A., \& Hallen, J. M. (2009). Markov chain modelling of pitting corrosion in underground pipelines. Corrosion Science, 51, $2197-$ 2207. doi:j.corsci.2009.06.014

Castro, I. T., Caballé, N. C., \& Pérez, C. J. (2015). A condition-based maintenance for a system subject to multiple degradation processes and external shocks. International Journal of Systems Science, 46(9), 16921704. doi:10.1080/00207721.2013.828796

Cox, D. R., \& Miller, H. D. (1965). The theory of stochastic processes. London, UK: Methuen \& Co. Ltd,.

Frangopol, D. M., Kallen, M. J., \& Van Noortwijk, J. M. (2004). Probabilistic models for life-cycle performance of deteriorating structures: review and future directions. Progress in Structural Engineering and Materials, 6(4), 197-212. doi:10.1002/pse.180

Hong, H. P. (1999). Application of the stochastic process to pitting corrosion. Corrosion, 55(1), 10-16. doi:10.5006/1.3283958

Imanian, A., \& Modarres, M. (2017). A thermodynamic entropy-based damage assessment with applications to prognostics and health management. Structural Health Monitoring. doi:10.1177/1475921716689561

Maes, M. A., Dann, M. R., Breitung, K. W., \& Brehm, E. (2008). 6th International Probabilistic Workshop. Darmstadt, Germany.

Maes, M. A., Faber, M. H., \& Dann, M. R. (2009). Hierarchical modeling of pipeline defect growth subject to ILI uncertainty. Proceedings of the ASME 28th International Conference on Ocean, Offshore and Arctic Engineering, (pp. OMAE2009-79470). Honolulu, Hawaii.

Melchers, R. E. (2004). Pitting corrosion of mild steel in marine immersion environment-Part 2: Variability of maximum pit depth. Corrosion, 937-944. doi:10.5006/1.3287827

Modarres, M., \& Nuhi, M. (2010, January 6). Assessment of the integrity of pipelines subject to corrosion-fatigue, pitting corrosion, creep and stress corrossion carcking. 1st Annual PI Partner Schools Research Workshop. Abu Dhabi, U.A.E. 
Nešić, S. (2007). Key issues related to modelling of internal corrosion of oil and gas pipelines-A review. Corrosion Science, 49(12), 4308-4338. doi:10.1016/j.corsci.2007.06.006

Nuhi, M., Seer, T. A., Al Tamimi, A. M., Modarres, M., \& Seibi, A. (2011). Reliability analysis for degradation effects of pitting corrosion in carbon steel pipes. Procedia Engineering(10), 1930-1935. doi:10.1016/j.proeng.2011.04.320

Nyborg, R. (2010). Co2 Corrosion Models For Oil And Gas Production Systems. Corrosion 2010 NACE International.

Nyborg, R. (2010). CO2 corrosion models for oil and gas production systems. Corrosion. San Antonio, Texas: NACE International.

Ossai, C. I., Boswell, B., \& Davies, I. J. (2015). Predictive modelling of internal pitting corrosion of aged nonpiggable pipelines. Journal of The Electrochemical Society, 162(6), C251-C259.

Papavinasam, S. (2013). Corrosion control in the oil and gas industry. Elsevier Inc.

Papavinasam, S., Revie, R. W., Friesen, W. I., Doiron, A., \& Panneerselvan, T. (2006). Review of models to predict internal pitting corrosion of oil and gas pipelines. Corrosion Reviews, 24(3-4), 173-230.

Provan, J. W., \& Rodriguez III, E. S. (1989). Part I: Development of a Markov description of pitting corrosion. Corrosion, 45(3), 178-192. doi:10.5006/1.3577840

Rabiei, E., Droguett, E. L., Modarres, M., \& Amiri, M. (2015). Damage precursor based structural health monitoring and damage prognosis framework. Safety and Reliability of Complex Engineered Systems, (pp. 2441-2449).

Romanoff, M. (1957). Underground corrosion. Washington, DC: US Government Printing Office.

Ross, S. (1996). Stochastic processes. John Wiley \& Sons,Inc.

Shibata, T. (1996). Statistical and stochastic approaches to localized corrosion. Corrosion, 52(11), 813-830. doi:10.5006/1.3292074

Strutt, J. E., Nicholls, J. R., \& \& Barbier, B. (1985). The prediction of corrosion by statistical analysis of corrosion profiles. Corrosion science, 305-315. doi:10.1016/0010-938X(85)90109-X

Tarantseva, K. (2010). Models and methods of forecasting pitting corrosion. Protection of metals and physical chemistry of surfaces, 46(1), 139-147. doi:10.1134/S2070205110010211

Tarantseva, K. (2010). Models and methods of forecasting pitting corrosion. Protection of metals and physical chemistry of surfaces, 46(1), 139-147.

Tsui, K. L., Chen, N., Zhou, Q., Hai, Y., \& Wang, W. (2015). Prognostics and health management: A review on data driven approaches. Mathematical Problems in Engineering. doi:10.1155/2015/793161
Valor, A., Caleyo, F., Alfonso, L., Rivas, D., \& Hallen, J. M. (2007). Stochastic modeling of pitting corrosion: a new model for initiation and growth of multiple corrosion pits. Corrosion science, 49(2), 559-579. doi:10.1016/j.corsci.2006.05.049

Valor, A., Caleyo, F., Alfonso, L., Velázquez, J. C., \& Hallen, J. M. (2013). Markov chain models for the stochastic modeling of pitting corrosion. Mathematical Problems in Engineering. doi:10.1155/2013/108386

Van Noortwijk, J. M. (2009). A survey of the application of gamma processes in maintenance. Reliability Engineering and System Safety, 94, 2-21. doi:10.1016/j.ress.2007.03.019

Velázquez, Caleyo, F., Valor, A., \& Hallen, J. (2009). Predictive model for pitting corrosion in buried oil and gas pipelines. Corrosion, 65(5), 332-342. doi:10.5006/1.3319138

Zhang, S., \& Zhou, W. (2013). System reliability of corroding pipelines considering stochastic process-based models for defect growth and internal pressure. International Journal of Pressure Vessels and Piping, 111(112), 120-130. doi:10.1016/j.ijpvp.2013.06.002

Zhang, S., \& Zhou, W. (2014). Bayesian dynamic linear model for growth of corrosion defects on energy pipelines. Reliability Engineering \& System Safety, 128, 24-31. doi:10.1016/j.ress.2014.04.001

Zhang, S., Zhou, W., \& Qin, H. (2013). Inverse Gaussian process-based corrosion growth model for energy pipelines considering the sizing error in inspection data. Corrosion Science, 73, 309-320. doi:10.1016/j.corsci.2013.04.020 